Poincare Journal of Analysis $\&$ Applications

Vol. 2018 (1), 25-33

(C) Poincare Publishers

OINCAR

UBLISH $2 \overline{\overline{R S}}$

\title{
Some results on the existence of frames in Banach spaces
}

\author{
SHAH JAHAN ${ }^{\dagger}$ AND VARINDER KUMAR
}

$\begin{array}{lll}\text { Date of Receiving } & : & 05.04 .2017 \\ \text { Date of Revision } & : & 06.10 .2017 \\ \text { Date of Acceptance } & : & 19.01 .2018\end{array}$

\begin{abstract}
The notion of $\mathcal{J}$-frames in Banach spaces has been defined and studied. A necessary and sufficient condition for the existence of a $\mathcal{J}$-frame in a Banach space has been given. Also, a sufficient condition, in terms of $\mathcal{J}$-frame, the existence of bounded approximation property in a Banach space has been given. Further, we study the interplay between the existence of retro Banach frames in conjugate Banach spaces and the existence of a support cone. Finally, we have given conditions for the existence of a retro Banach frame in the conjugate space of a Banach space with Fréchet differentiable norm.
\end{abstract}

\section{Introduction}

Dennis Gabor [12] in 1946 introduced a fundamental approach to signal decomposition in terms of elementary signals. Later, in 1952, While addressing some deep problems in non-harmonic Fourier series, Duffin and Schaeffer [9] abstracted Gabors method to define frames for Hilbert spaces. These ideas did not generate much interest outside of non-harmonic Fourier series and signal processing for more than three decades until Daubechies, Grossmann and Meyer [8] reintroduced frames. After this land mark paper the theory of frames begin to be studied widely and found new applications to wavelet and Gabor transforms in which frames played an important role. Frames are generalizations of orthonormal bases in Hilbert spaces. The main property of frames which makes them useful is their redundancy.

Representation of signals using frames is advantageous over basis expansions in a variety of practical applications in pure and applied mathematics. The theory of frame has grown rapidly in the past one decade in view of wide range of its applications in signal processing, wireless communications(CDMA system) image processing, differential equations, filter banks, geophysics, quantum computing, wireless sensor network, multiple-antenna code design and many more. The reason for such wide applications is that frames provide both great liberties in design of vector space. For a nice and

2010 Mathematics Subject Classification. 42C15, 46B15, 46B20.

Key words and phrases. Banach frames, Retro Banach frames, $\mathcal{J}$-frames, support cone.

Communicated by: F. A. Shah

${ }^{\dagger}$ Corresponding author. 